\title{
Bloodstream Infection in the Paediatric Cancer Patients: Bacteriological Profile and Drug Resistance Patterns in Shenzhen, China
}

\author{
Sandip Patil ${ }^{1,2}$, Bruno S Lopes ${ }^{3}$, Hongyu Chen ${ }^{4}$, Lian $\mathrm{Ma}^{2}$ and Feiqiu Wen ${ }^{1,2^{*}}$ \\ ${ }^{1}$ Department of Haematology and Oncology, Shenzhen Children's Hospital, District Futian, Shenzhen, China \\ ${ }^{2}$ Paediatric Research Institute, Shenzhen Children's Hospital, District Futian, Shenzhen, China \\ ${ }^{3}$ Department of Medical Microbiology, School of Medicine, Medical Sciences and Nutrition, Aberdeen University, China \\ ${ }^{4}$ Department of Laboratory Medicine, Shenzhen Children's Hospital, District Futian, Shenzhen, China
}

*Corresponding author: Feiqiu Wen, Department of Haematology and Oncology, Shenzhen Children's Hospital, District Futian, Shenzhen, China, Tel: +86-18938690333; Fax: +86-75583009888; E-mail: fwen62@126.com

Received date: December 18, 2018; Accepted date: January 08, 2019; Published date: January 17, 2019

Copyright: ( 2019 Patil S, et al. This is an open-access article distributed under the terms of the Creative Commons Attribution License, which permits unrestricted use, distribution, and reproduction in any medium, provided the original author and source are credited.

\begin{abstract}
Bloodstream Infections (BSI) remain a main cause of death in pediatric cancer patients. We present the first report on the microbiological profile of bacteremia and drug resistance patterns in Shenzhen, China. We examined the types of bloodstream infectious agents and their antimicrobial susceptibility pattern in the pediatric oncology patients, from Shenzhen Children's Hospital from January 2016 to October 2018. Acinetobacter baumannii (12.82\%), Klebsiella pneumoniae (12.82\%) and Staphylococcus epidermidis $(34.61 \%)$ were highly prevalent in cancer patients with bloodstream infection. Extended-spectrum beta-lactamase-producing Escherichia coli and Klebsiella pneumoniae were also predominant. $29 \%$ of isolates were resistant to more than two class of antibiotics so call them as multi-drug resistant isolates.
\end{abstract}

Keywords: Multi-drug resistant bacterial infection; ESBL; Cancer

\section{Letter to the Editor}

Bloodstream Infections (BSI) remains a key cause of morbidity and mortality in patients undergoing cancer treatment [1]. Antimicrobial Resistance (AMR) pattern of pathogens causing BSI, significantly differ in developed and developing countries [2]. In this study, we focused on the trends of etiological agents causing BSI and determined the antimicrobial susceptibility pattern of bacteria isolated from pediatric oncology patients in Shenzhen, China for the same. A total of 607 nonrepetitive isolates were collected by the Department of Haematology and Oncology, Shenzhen Children's Hospital during January 2016October 2018. A total of 607 isolates recovered from all specimens includes blood, pus, urine, and catheter-associated secretions but our concerns with $78(12.85 \%)$ isolate from a bloodstream infection. We studied total $78(12.85 \%)$ isolates recovered from samples with BSI, comprising of $17(2.8 \%), 40(6.5 \%)$ and $21(3.4 \%)$ isolates from the year 2016, 2017 and 2018 respectively. The Horasan and his co-worker reported BSI to be $21.4 \%$ in adult cancer patients which is higher than our observation [3]. In our study, $40(51.28 \%)$ isolates were recovered from a female, while $38(48.78 \%)$ were from male patients. The present study revealed the frequency of occurrence of Gram-negative bacteria to be about $47.43 \%$ ( $n=37)$ which was slightly lower than that of Grampositive bacteria with $52.57 \% \quad(n=41)$. Our observations are comparable with a published study conducted in Egypt [4]. All recovered isolates were confirmed by the API20 system in our clinical laboratory. During this period, blood samples were accounted for pathogenic bacteria Staphylococcus epidermidis 34.61\% $(\mathrm{n}=27)$, followed by Acinetobacter baumannii $12.82 \% \quad(\mathrm{n}=10)$, Klebsiella pneumoniae $12.82 \%(\mathrm{n}=10)$, Pseudomonas aeruginosa $10.25 \%(\mathrm{n}=8)$, Staphylococcus hemolyticus and Staphylococcus hominis each $8.9 \%$ $(\mathrm{n}=7)$, Escherichia coli $5.12 \%(\mathrm{n}=4)$, Comamonas testosteroni $3.8 \%$ $(\mathrm{n}=3)$ and Enterobacter cloacae 2.5\% $\quad(\mathrm{n}=2)$. Antimicrobial susceptibility was determined by broth dilution method and breakpoint was determined as per CSLI standard. In addition, Extended-Spectrum Beta-Lactamase (ESBL) production was confirmed by using combination disc test. The test was performed by using Disc of both cefotaxime and ceftazidime, alone and in combination with clavulanic acid. The Gram-Negative pathogens were resistant to Amikacin, Ampicillin, Aztreonam, Ceftriaxone, Cefotetan, Cefazolin, Nitrofurantoin, Ampicillin-sulbactam, Trimethoprim, Tobramycin, and Piperacillin but sensitive to Ertapenem, Cefepime, Gentamicin, Imipenem, Levofloxacin and Ciprofloxacin (Figure 1). Gram-positive bacteria were resistant to the commonly used first-line of antibiotics (Figure 2). Antimicrobial susceptibility results showed that all Acinetobacter baumannii and Klebsiella pneumoniae isolates were resistant to carbapenem antibiotics. These multi-drug resistant pathogens are considered to be superbugs which may pose a serious threat to human health, especially in cancer patients. Such multi-drug resistant Klebsiella pneumoniae was reported from bloodstream infection in Tunisia [5]. We confirmed a rarely observed Comamonas testosteroni in bloodstream infection which showed $100 \%$ resistance to commonly used antibiotics. ESBL production was confirmed in two Escherichia coli and two Klebsiella pneumoniae isolates. The Staphylococcus species were resistant to Trimethoprim sulfamethoxazole followed by Nitrofurantoin, Oxacillin, Erythromycin, Tetracycline, Clindamycin, and Ciprofloxacin but sensitive to Quinupristin-dalfopristin, Moxifloxacin, Tigecycline, and Vancomycin (Figure 2). In this study, we evaluated the antimicrobial resistance pattern of Gram-positive and Gram-negative pathogens causing BSI, on the basis of phenotypic expression. Acinetobacter baumannii, Klebsiella pneumoniae, and Staphylococcus epidermidis were highly prevalent in the paediatric cancer patients with BSI. High microbial resistance was detected in causative agents of bloodstream infection in the paediatric patients with cancer. Our findings, bacteriological 
Citation: Patil S, Lopes BS, Chen H, Ma L, Wen F (2019) Bloodstream Infection in the Paediatric Cancer Patients: Bacteriological Profile and Drug Resistance Patterns in Shenzhen, China. Clin Microbiol 8: 324. doi:10.4172/2327-5073.1000324

Page 2 of 2

profile and antimicrobial resistance pattern of BSI in the paediatric cancer patients, can help to determine the factors governing these infections, to antibiotic treatment policy and to improve the infection control measures. We suggest that the continual monitoring of bloodstream infections is highly essential.

\begin{tabular}{|c|c|c|c|c|c|c|c|c|c|c|c|c|c|c|c|c|c|c|c|}
\hline \multirow[t]{2}{*}{ Bacteria } & \multirow{2}{*}{$\begin{array}{c}\text { No. } \\
\text { isolates }\end{array}$} & \multicolumn{18}{|c|}{ Drug-resistant isolates, \% } \\
\hline & & AMK & AMP & ATM & CAZ & CIP & CRO & $\mathrm{CT}$ & CZO & ETP & FEP & GEN & IMP & LVX & NIT & SAM & SXT & TOB & TZP \\
\hline $\begin{array}{l}\text { Acinetobacter } \\
\text { baumannil }\end{array}$ & 10 & 100 & 100 & 100 & 100 & 0 & 100 & 100 & 100 & $N / D$ & 0 & 0 & 0 & 0 & 100 & 10 & 20 & 0 & $\mathrm{~N} / \mathrm{D}$ \\
\hline $\begin{array}{l}\text { Klebsiella } \\
\text { pneumoniae }\end{array}$ & 10 & 20 & 100 & 30 & 4 & 30 & 30 & 10 & 30 & 10 & 30 & 40 & 10 & 20 & 100 & 100 & 30 & 40 & 20 \\
\hline $\begin{array}{l}\text { Pseudomonas } \\
\text { aeruginosa }\end{array}$ & 8 & 37.5 & 100 & $\mathrm{~N} / \mathrm{D}$ & 100 & 0 & 100 & 100 & 100 & $N / D$ & 37.5 & 37.5 & 25 & 0 & 100 & 100 & 100 & 12.5 & 75 \\
\hline $\begin{array}{l}\text { Escherichia } \\
\text { Coli }\end{array}$ & 4 & 0 & 0 & 0 & 25 & 50 & 50 & 100 & 100 & 0 & 0 & 0 & 0 & 100 & 0 & 75 & 100 & 50 & 100 \\
\hline $\begin{array}{l}\text { Comonas } \\
\text { testosteroni }\end{array}$ & 3 & 100 & 100 & 100 & 0 & 0 & 100 & 100 & 100 & $\mathrm{~N} / \mathrm{D}$ & 100 & 100 & 0 & 0 & 100 & 100 & 100 & 100 & 100 \\
\hline $\begin{array}{l}\text { Enterobacter } \\
\text { cloacae }\end{array}$ & 2 & 100 & 100 & 2 & 0 & 0 & 0 & 0 & 100 & 0 & 0 & 0 & 0 & 0 & 100 & 100 & 0 & 0 & 0 \\
\hline
\end{tabular}

-AMK, Amikacin; AMP, Ampicilin; ATM, Aztreonam; CAZ, Ceftazidime; CIP, Ciprofloxacin; CRO, Ceftriaxone; CTT, Cefotetan; CZO, Cefazolin; ETP, Ertapenem; FEP, Cefepime; GEN, Gentamicin; IMP, Imipenem; LVX, Levofloxacin; NIT, Nitrofurantoin; SXT, Trimethoprim; FEP, Cefepime; SAM, Ampicilin-sulbactam; TOB, Tobramycin; TZP, Piperacillin

Figure 1: Antimicrobial Susceptibility of Gram-negative bacteria from a paediatric bloodstream infection.

\begin{tabular}{|c|c|c|c|c|c|c|c|c|c|c|c|c|c|c|c|c|c|}
\hline \multirow[t]{2}{*}{ Bacteria } & \multirow[t]{2}{*}{ No. Isolates } & \multicolumn{16}{|c|}{ Drug-resistant isolates, \% } \\
\hline & & CIP & $\mathrm{CLI}$ & ERY & GEN & LNZ & LVX & MXF & NIT & OXA & PEN & QDA & RIF & SXT & TC & TGC & VAN \\
\hline $\begin{array}{l}\text { Staphylococcus } \\
\text { epidermidis }\end{array}$ & 27 & 14.8 & 29.6 & 46 & 25.9 & 7.4 & 11.11 & 0 & 96.2 & 59.25 & 0 & 0 & 11.11 & 100 & 37 & 0 & 0 \\
\hline $\begin{array}{l}\text { Staphylococcus } \\
\text { hemolyticus }\end{array}$ & 7 & 85.7 & 85.7 & 100 & 85.7 & 100 & \begin{tabular}{|l|}
6 \\
85.7
\end{tabular} & \begin{tabular}{|l|}
6 \\
85.7
\end{tabular} & 100 & 85.7 & 0 & 0 & 85.7 & 85.7 & 85.7 & 0 & 0 \\
\hline $\begin{array}{l}\text { Staphylococcus } \\
\text { hominis }\end{array}$ & 7 & 0 & 14.28 & 57.14 & 14.28 & 71.42 & 0 & 0 & 71.42 & 14.28 & 2 & 0 & 14.28 & 100 & 57.14 & 0 & 0 \\
\hline
\end{tabular}

${ }^{*}$ CIP, Ciprofloxacin; CU, Clindamycin; ERY, Erythromycin; GEN, Gentamicin; LNZ, Linezolid; LVX, Levofloxacin; Mxf, Moxifloxacin; NIT, Nitrofurantoin; OXA,

Oxacillin; PEN, Penicillin; QDA, Quinupristin-dalfopristin; RIF, Rifampin; SXT, Trimethoprim-sulfomethoxazole; TC, Tetracycline; TGC, Tigecycline; VAN,

Vancomycin.

Figure 2: Antimicrobial Susceptibility of Gram-positive bacteria from a paediatric bloodstream infection.

\section{Funding}

This work was supported by a grant from Sciences and Technology Project from sciences technology and innovation committee of Shenzhen municipality (Grant No. JCYJ20170817170110940).

\section{References}

1. Montassier E, Batard E, Gastinne T, Potel G (2013) Recent changes in bacteraemia in patients with cancer: a systematic review of epidemiology and antibiotic resistance. Eur J Clin Microbiol Infect Dis 32: 841-850.

2. Dat Q, Vu N, Nguyen H, Nguyen T, Hoang B, et al. (2017) Bacterial bloodstream infections in a tertiary infectious disease's hospital in
Northern Vietnam: etiology, drug resistance, and treatment outcome. BMC Infect Dis 17: 493.

3. Horasan E, Ersoz G, Tombak A, Tiftik N, Kaya A (2011) Bloodstream infections and mortality-related factors in febrile neutropenic cancer patients. Med Sci Monit 17: 304-309.

4. Hadir A, Safaa S, Mohamed E, Manar M, Lobna S (2015) Update on healthcare-associated bloodstream infection in febrile neutropenic pediatric oncology patient. Journal of Cancer Therapy 6: 503-510.

5. Farah Ben T, Anis R, Yosra C, Wafa A (2018) Epidemiology and molecular characterization of colistin-resistant Klebsiella pneumoniae isolates from immunocompromised patients in Tunisia. Int Journal of Antimicrobial Agents 52: 861-865. 